OPEN ACCESS

Edited by:

Djoher Nora Abrous,

Institut des Neurosciences de

Bordeaux, France

Reviewed by:

Therese M. Jay,

Institut National de la Santé et de la

Recherche Médicale, France

Phillip R. Zoladz,

Ohio Northern University, USA

*Correspondence:

Linda L. Chao

linda.chao@ucsf.edu

Received: 27 September 2015 Accepted: 19 November 2015 Published: 02 December 2015

Citation:

Chao LL, Tosun D, Woodward SH, Kaufer D and Neylan TC (2015) Preliminary Evidence of Increased Hippocampal Myelin Content in Veterans with Posttraumatic Stress Disorder.

Front. Behav. Neurosci. 9:333 doi: 10.3389/fnbeh.2015.00333

\section{Preliminary Evidence of Increased Hippocampal Myelin Content in Veterans with Posttraumatic Stress Disorder}

\author{
Linda L. Chao ${ }^{1,2,3 *}$, Duygu Tosun ${ }^{1,2}$, Steven H. Woodward ${ }^{4}$, Daniela Kaufer ${ }^{5,6,7}$ \\ and Thomas C. Neylan ${ }^{3,8}$ \\ ${ }^{1}$ Center for Imaging of Neurodegenerative Diseases, Veterans Affairs Medical Center, San Francisco, CA, USA, ${ }^{2}$ Department \\ of Radiology and Biomedical Imaging, University of California, San Francisco, CA, USA, ${ }^{3}$ Department of Psychiatry, \\ University of California, San Francisco, CA, USA, ${ }^{4}$ Dissemination and Training Division, National Center for PTSD, VA Palo \\ Alto Health Care System, CA, USA, ${ }^{5}$ Helen Wills Neuroscience Institute, University of California, Berkeley, CA, USA, \\ ${ }^{6}$ Department of Integrative Biology, University of California, Berkeley, CA, USA, ${ }^{7}$ Canadian Institute for Advanced Research \\ (CIFAR), Toronto, ON, Canada, ${ }^{8}$ Mental Health Services, Veterans Affairs Medical Center, San Francisco, CA, USA
}

Recent findings suggest the formation of myelin in the central nervous system by oligodendrocytes is a continuous process that can be modified with experience. For example, a recent study showed that immobilization stress increased oligodendrogensis in the dentate gyrus of adult rat hippocampus. Because changes in myelination represents an adaptive form of brain plasticity that has a greater reach in the adult brain than other forms of plasticity (e.g., neurogenesis), the objective of this "proof of concept" study was to examine whether there are differences in myelination in the hippocampi of humans with and without post-traumatic stress disorder (PTSD). We used the ratio of T1-weighted/T2-weighted magnetic resonance image (MRI) intensity to estimate the degree of hippocampal myelination in 19 male veterans with PTSD and 19 matched trauma-exposed male veterans without PTSD (mean age: $43 \pm 12$ years). We found that veterans with PTSD had significantly more hippocampal myelin than trauma-exposed controls. There was also found a positive correlation between estimates of hippocampal myelination and PTSD and depressive symptom severity. To our knowledge, this is the first study to examine hippocampal myelination in humans with PTSD. These results provide preliminary evidence for stress-induced hippocampal myelin formation as a potential mechanism underlying the brain abnormalities associated with vulnerability to stress.

Keywords: myelin, hippocampus, post-traumatic stress disorder, imaging, plasticity

\section{INTRODUCTION}

It is well known that our brains are constantly remodeling neuronal pathways as we learn and experience the world around us. Exciting recent findings suggest that this remodeling process is not just a property of neurons, but that oligodendrocytes, the main myelin forming cells in the central nervous system, and their precursors are also adapting and changing with experience (Young et al., 2013; Gibson et al., 2014; Long and Corfas, 2014). Furthermore, 
there is evidence that the formation of myelin is a continuous and modifiable process that contributes to psychiatric disorders and other diseases that affect cognition (Fields, 2008). For example, a study that examined genes in prefrontal cortex of schizophrenic brains found 35 of 89 abnormally regulated genes were involved in myelination (Hakak et al., 2001).

Alterations in white matter tracts and myelin formation have been noted in numerous mental disorders, including posttraumatic stress disorder (PTSD; e.g., Daniels et al., 2013). There are also hints that stress may affect white matter through the regulation of oligodendrogenesis. For example, glucocorticoid stress hormones are potent inducers of pro-oligodendrogenic transcription factors and increase oligodendrogenesis (Almazan et al., 1986; Barres et al., 1994; Mann et al., 2008) and myelination (Kumar et al., 1989; Tsuneishi et al., 1991; Masters et al., 1994; Zhu et al., 1994; Cheng and de Vellis, 2000; Desarnaud et al., 2000) in oligodendrocyte precursor cell culture. Glucocorticoids have also been shown to dysregulate myelination in utero (Antonow-Schlorke et al., 2009). Recently, Chetty et al. (2014) reported that immobilization stress increased oligodendrogensis in the dentate gyrus of adult rat hippocampus. Because changes in myelination represents a form of adaptive brain plasticity that has a far greater reach in the adult brain (Young et al., 2013; Gibson et al., 2014; Long and Corfas, 2014) than other forms of plasticity (e.g., adult neurogenesis), the present study sought to examine whether vulnerability to posttraumatic stress is similarly associated with myelin dysregulation in humans.

Although myelinated axons are located predominately in white matter, they also exist in gray matter (Hildebrand et al., 1993; Nieuwenhuys, 2013). Because myelin co-varies with T1-weighted (T1w) and T2-weighted (T2w) image intensity from magnetic resonance images (MRI) in opposite directions (Yoshiura et al., 2000), this makes it possible to estimate the degree of myelination in the human brain in vivo using ratio of $\mathrm{T} 1 \mathrm{w} / \mathrm{T} 2 \mathrm{w}$ image intensity Glasser and Van Essen (2011). In a validation of this method, Bock et al. (2009, 2011) compared T1 and T1w/T2w image intensity maps to myelin stains in the marmoset monkey. They found that the myelin features of various cortical areas (e.g., the primary visual, auditory, somatosensory, and motor cortices) identified non-invasively with $\mathrm{T} 1 \mathrm{w} / \mathrm{T} 2 \mathrm{w}$ image intensity corresponded well with myelin stained sections in the same animals. Another validation study showed that a post-mortem T1w/T2w map of human somatosensory cortex had an intensity border closely aligned with the myeloarchitectonically and cytoarchitectonically defined border between areas 4 and $3 \mathrm{a}$ as seen in histological stains of the same piece of tissue (Geyer et al., 2011).

The objective of this "proof of concept" study was to use the ratio of $\mathrm{T} 1 \mathrm{w} / \mathrm{T} 2 \mathrm{w}$ image intensity to examine whether there are differences in the degree of hippocampal myelination in individuals with and without PTSD. Based on the findings of Chetty et al. (2014), who focused on the hippocampus because of its role in regulating memory and emotion and because it is the site of some of the most significant neurological impact of trauma (Sherin and Nemeroff, 2011), we hypothesized that veterans with PTSD would have greater hippocampal myelination than veterans without PTSD.

\section{MATERIALS AND METHODS}

\section{Participants}

Magnetic resonance imaging data from 38 males veterans (mean age: $43 \pm 12$ years) were examined for this study. All participants provided written informed consent approved by the University of California at San Francisco and the Veterans Administrations Committees on Human Research.

Twenty veterans included in the current analysis (8 PTSD+, 12 PTSD-) were recruited as part of a larger study investigating the effects of service in the Persian Gulf War on brain structure and brain function. Eighteen other veterans (11 PTSD+, 7 PTSD-) were recruited from the outpatient mental health clinic of the San Francisco Veterans Affairs Medical Center and by advertising in the community. All veterans participating in the study were evaluated by a $\mathrm{Ph} . \mathrm{D}$. level clinical interviewer using the Structured Clinical Interview for DSM-IV Diagnosis (SCID, First et al., 1995) the Clinician Administered PTSD Scale (CAPS, Blake et al., 1995) and an interview version of the Life Stressor Checklist-Revised (Wolfe et al., 1996) to determine exposure to traumatic events. The Life Stressor Checklist-Revised assesses 21 stressful life events (e.g., experiencing or witnessing serious accidents, illnesses, sudden death, and physical and sexual assault). The SCID was used to diagnose current major depressive disorder (MDD) and to rule out individuals with a lifetime history of psychotic or bipolar disorders and alcohol abuse or dependance within the previous 12 months and drug abuse or dependance within the previous six months. Other exclusion criteria were neurological illness, head trauma with loss of consciousness greater than $10 \mathrm{~min}$, medical disorders affecting brain function, and conditions ineligible for MRI.

All subjects with PTSD had traumatic exposure related to combat. The trauma histories of the subjects without PTSD included 16 individuals who served in combat, two who experienced traumatic accidents, and one who experienced a traumatic physical assault. The groups included a mixture of Gulf War (82\%), Iraq (32\%), Afghanistan (4\%), Beirut (4\%), Vietnam (4\%), and multiple (7\%) theater exposure. Three subjects with PTSD were taking antidepressant medications, two subjects (1 PTSD+, 1 PTSD-) were on antiepileptic medication for neuropathic pain, while four subjects (2 PTSD+, 2PTSD-) had been prescribed atypical antipsychotic medication (i.e., Ziprasidone) for anxiety and depression. Eleven subjects (9 PTSD+, 2 PTSD-) had a diagnosis of current major depressive disorder. Twelve subjects (7 PTSD+, 5 PTSD-) reported a history of alcohol abuse or dependance. Five subjects (2 PTSD+, 3 PTSD-) reported a history of drug abuse or dependance. On average, the episodes of alcohol/drug abuse/dependance occurred 17.6 years (range, 1-42 years) before the study. None of the episodes occurred within a year of the study. Eighteen subjects (8 PTSD+, 10 PTSD-) had Gulf War Illness (GWI) according to the Centers for Disease Control and 
Prevention (CDC) case definition (Fukuda et al., 1998). Six subjects (3 PTSD+, 3 PTSD-) had suspected exposure to lowlevels of sarin as determined by information obtained from the Directorate for Deployment Health Support of the Special Assistant to the Under Secretary of Defense (Personnel and Readiness) for Gulf War Illness Medical Readiness and Military Deployments. US demolition operations at the Khamisiyah ammunition point (case narrative) (2002). The demographics and clinical characteristics of the participants are summarized in Table 1.

\section{Measures}

We used the total CAPS score to assess current PTSD symptom severity, the Hamilton Depression rating scale (HAM-D; Hamilton, 1967) to assess depressive symptomology.

\section{MRI Acquisition and Data Processing}

Magnetic resonance imaging scans were acquired at the Center for Imaging of Neurodegenerative Diseases at the San Francisco Veterans Affairs Medical Center using a Bruker/Siemens MedSpec 4T MRI system (Bruker BioSpin, Ettlingen, Germany) equipped with an 8-channel array receiver coil. The MRI scan protocol consisted of a volumetric T1-weighted magnetization prepared gradient echo sequence (repetition time, $2300 \mathrm{~ms}$; time following inversion pulse, $950 \mathrm{~ms}$; echo time, $4 \mathrm{~ms} ; 7^{\circ}$ excitation pulses; $1 \mathrm{~mm}$ isotropic resolution) and a volumetric T2-weighted turbospin echo sequence (repetition time, 3400 ms; echo time, 403 ms; 109 echoes per k-space segment with variable flip angles; Slice Turbo factor $=2, \mathrm{FOV}=256 \mathrm{~mm}$ $\times 224 \mathrm{~mm} \times 176 \mathrm{~mm}, 1 \mathrm{~mm}$ isotropic resolution, and 176 continuous sagittal slices), acquired during the same scanning session.

TABLE 1 | Sample characteristics.

\begin{tabular}{|c|c|c|}
\hline & PTSD- & PTSD+ \\
\hline $\mathrm{N}$ & 19 & 19 \\
\hline Age (in years) & $43.0(13.8)$ & $42.7(10.7)$ \\
\hline No. (\%) Caucasian & $10(53 \%)$ & $12(63 \%)$ \\
\hline Education (in years) & $14.8(1.8)$ & $13.7(1.7)$ \\
\hline CAPS & $10.3(9.5)$ & $59.4(15.8)^{* *}$ \\
\hline HAM-D & $5.0(6.1)$ & $12.3(5.5)^{* *}$ \\
\hline No. (\%) with current MDD & $2(11 \%)$ & $9(47 \%)^{*}$ \\
\hline No. (\%) on psychotropic medication & $3(16 \%)$ & $6(32 \%)$ \\
\hline No. (\%) with history of alcohol abuse/dependance & $5(26 \%)$ & $7(37 \%)$ \\
\hline No. (\%) with history of drug abuse/dependance & $3(16 \%)$ & $2(11 \%)$ \\
\hline No. (\%) Gulf War veterans & $13(68 \%)$ & $11(58 \%)$ \\
\hline No. (\%) with Gulf War Illness ${ }^{a}$ & $10(53 \%)$ & $8(42 \%)$ \\
\hline No. (\%) with suspected sarin exposure ${ }^{b}$ & $3(16 \%)$ & $3(16 \%)$ \\
\hline
\end{tabular}

Means (SD) or number (\%) reported. Abbreviations: PTSD, post-traumatic stress disorder, CAPS, Clinician Administered PTSD Scale, MDD, major depressive disorder, HAM-D, Hamilton Depression Scale. Significantly different from PTSDat ${ }^{*} p<0.05,{ }^{* *} p<0.001$. a according to Centers for Disease Control and Prevention case definition (Fukuda et al., 1998). ${ }^{b}$ according to the Directorate for Deployment Health Support of the Special Assistant to the Under Secretary of Defense (Personnel and Readiness) for Gulf War IIIness Medical Readiness and Military Deployments. US demolition operations at the Khamisiyah ammunition point (case narrative) (2002).
The T2w images were registered to the T1w images using FSL's FLIRT (Jenkinson et al., 2002) with six parameters (rigid body) and the mutual information cost function. Next, the T2w images were resampled using the trilinear interpolation onto T1 imaging space. Finally, the T1w images were divided by the aligned T2w images to estimate myelin content. As proposed by Glasser and Van Essen (2011), T1w and T2w images were not preprocessed for the signal intensity bias related to the sensitivity profile of the radio frequency receiver coils before generating the ratio image. Assuming that the signal intensity bias was the same in both images, the signal intensity bias was mathematically canceled by the ratio operator.

Because we were interested in estimating the degree of myelination in the hippocampus, we did not perform surfacebased analyses of the T1w /T2w data as Glasser and Van Essen (2011) had done. Instead, we conducted a region of interest (ROI) analysis using the Freesurfer version 4.5 tissue boundary delineation of the hippocampus (Fischl et al., 2004). Specifically, we extracted T1w/T2w values from the T1w/T2w images, which were in $\mathrm{T} 1$ space, from the Freesurfer hippocampal ROI (see Figure 1). Because we did not have a priori hypotheses about laterality differences and because Chetty et al. (2014) did not report laterality differences, we averaged the estimates of left and right hippocampal myelin to reduce the number of measurements.

\section{Analyses}

Statistical analyses of the demographic, clinical, and T1w/T2w values were performed using IBM SPSS Statistics, version 23. Demographic, descriptive, and clinical characteristics were compared across the groups with student's $t$-test for continuous variables and Fisher's exact of independance for categorical variables. Because the Shaprio-Wilks test indicated that the variables of interest (hippocampal T1w/T2w ratio and CAPS) were not normally distributed, we used the nonparametric Mann-Whitney test to examine group differences in hippocampal myelin content and Spearman's Rank-Order correlation to examine the relationship between hippocampal myelin content and current PTSD symptoms severity (i.e., CAPS).

\section{RESULTS}

Table 1 summarizes the demographic and clinical characteristics of the 19 PTSD+ and the 19 PTSD- subjects. As expected, there were no significant group differences in age, years of education, race, history of alcohol and/or drug abuse/dependance, number of GW veterans, number of veterans with Gulf War Illness, or number of veterans with suspected sarin exposure. The PTSD+ group had higher CAPS $(t=11.62, d f=36, p<0.001)$ and HAM-D $(t=0.83, d f=36, p<0.001)$ scores and a higher incidence of current MDD ( $p=0.03$, Fisher's exact test).

There was a significant group difference in the estimate of hippocampal myelination ( $p=0.006$, Mann-Whitney $U$-test; see Figure 2). Bivariate correlations revealed significant positive correlations between estimates of hippocampal myelination, 


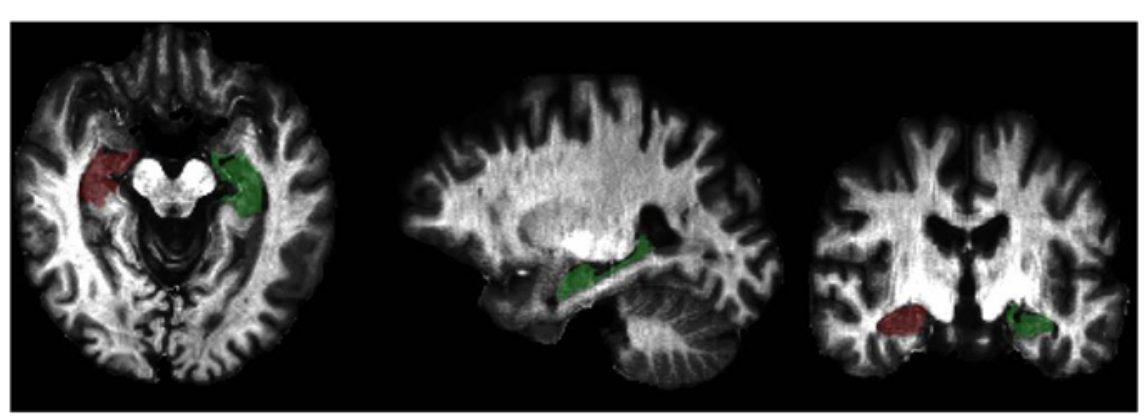

FIGURE 1 | Graphical representation of the Freesurfer hippocampal region of interest (ROI) overlaid on a T1w/T2w intensity map

CAPS (Spearman's $\rho=0.38, p=0.019$ ), and HAM-D (Spearman's $\rho=0.46, p=0.004$ ) scores.

\section{DISCUSSION}

To our knowledge, this is the first study to examine the degree of hippocampal myelination in humans with PTSD. We found that veterans with PTSD had a significantly greater degree of hippocampal myelination compared to matched, traumaexposed veterans without PTSD. The degree of hippocampal myelination was also significantly and positively correlated with current PTSD symptom severity. These findings, consistent with the recent report in rats by Chetty et al. (2014), suggest that vulnerability to traumatic stress may similarly dysregulate myelination in the human hippocampus.

Chetty et al. (2014) showed that stress stimulated the production of adult oligodendrocytes from neural stem cells in the rat dentate gyrus. It is unclear if a similar mechanism or if other mechanisms are responsible for the increased hippocampal myelin content that we observed in veterans with

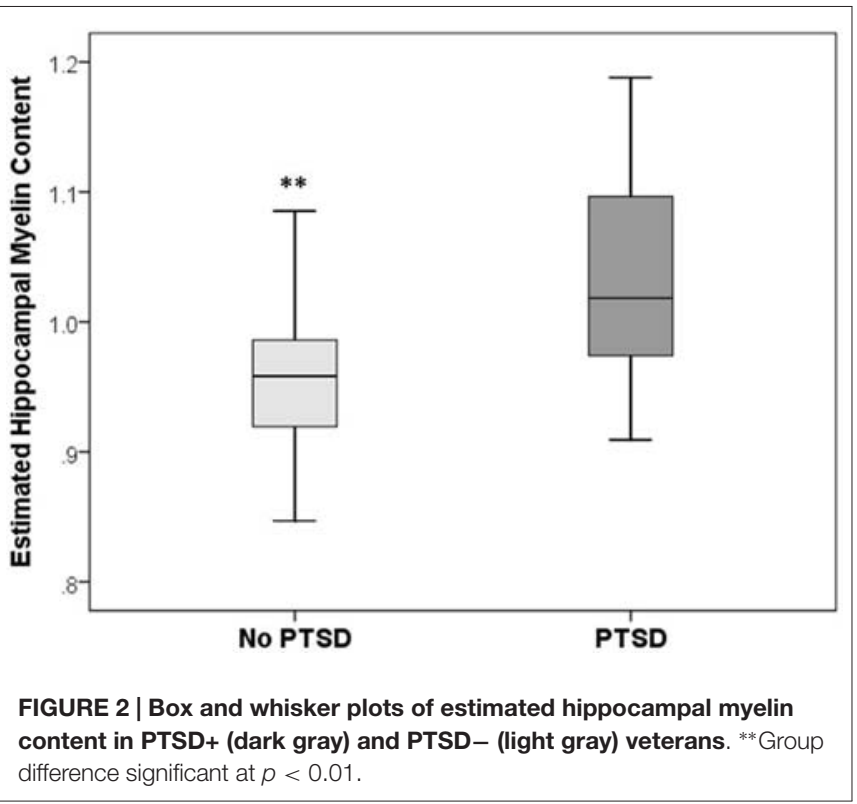

PTSD in the current study. For example, stress may promote myelin synthesis in pre-existing adult oligodendrocytes (Young et al., 2013). Alternatively, stress may stimulate the activity of oligodendrocyte precursor cells in the parenchyma and the subventricular zone. Whatever the mechanism, it is significant that we found hints of maladaptive myelin development in humans with vulnerability to stress because: (i) maladaptive myelin formation has been implicated in other psychiatric disorders (Fields, 2008); (ii) Changes in myelination represents a form of plasticity that has a greater reach in the brain regions implicated in PTSD (i.e., amygdala, anterior cingulate, insula, and orbitofrontal region (Rauch et al., 2006; Shin et al., 2006) than adult neurogenesis, which is limited to a small population of neuronal stem cells (Schoenfeld and Gould, 2012); and (iii) PTSD has been associated with white matter alterations (Daniels et al., 2013).

Although our results concern myelination in the hippocampus, a gray matter structure, as noted in the introduction, myelinated axons are located predominately in white matter. There are several white matter tracts associated with the hippocampus and medial temporal lobe (MTL): the performant path emerges from the entorhinal cortex and ends in the dentate gyrus in the hippocampus (Knowles, 1992). The fornix contains hippocampal projections to the mammillary bodies and anterior nuclei of the thalamus (Nowrangi and Rosenberg, 2015). The ventral cingulum bundle connects parahippocampal gyrus to the posterior cingulate cortex (Jones et al., 2013). Although it does not extend into the hippocampus, the uncinate fasciculus connects the anterior temporal lobe to lateral orbitofrontal cortex through a direct, monosynaptic, bidirectional pathway (Von Der Heide et al., 2013).

Diffusion tensor imaging (DTI) is an imaging technique that allows for the interrogation of the microstructural integrity of white matter (Le Bihan, 2003). A few DTI studies have attempted to investigate the performant pathway in vivo in patients with mild cognitive impairment (MCI), an intermediate stage between normal aging and Alzheimer's disease (AD; Morris et al., 2001), and AD. However, these studies were limited by low resolution and the inability to specifically identify performant pathway fibers (Kalus et al., 2006; Rogalski et al., 2009). Recently, Yassa et al. (2010) used ultrahigh-resolution microstructural DTI with submillimeter resolution to identify diffusion signals unique 
to the performant path. However, this technique has yet to be applied to populations with PTSD.

In a meta-analysis of seven whole-brain DTI studies of trauma-exposed adults, Daniels et al. (2013) identified significant decreases and increases in fractional anisotropy (FA), a DTI metric commonly used as a proxy measure for white matter integrity (Alexander et al., 2007), in the dorsal cingulum. The largest cluster in the right dorsal cingulum pertained to FA decreases; however, Daniels et al. (2013) also identified clusters of FA increases and FA decreases bilaterally in other sections of the dorsal cingulum. In a longitudinal study of treatmentrelated DTI differences in remitted and persistent PTSD patients and combat controls, Kennis et al. (2015) suggested that higher FA in the dorsal cingulum bundle may be an acquired feature of persistent PTSD that develops over time. Kennis et al. (2015) also found significant group by time interactions in the hippocampal (ventral) cingulum, fornix, and stria terminalis, which they suggested might reflect the differential effects that PTSD treatment may have on FA in these white matter tracts in remitted vs. persistent PTSD patients.

It is noteworthy that the Kennis et al. (2015) did not find significant overall effects of group or time in hippocampal/MTL white matter tracts. In fact, no DTI studies of populations affected by PTSD to date have reported significant DTI changes in hippocampal or MTL white matter tracts. In contrast, numerous DTI studies of patients at risk for AD (Mielke et al., 2012; Douaud et al., 2013) and MCI (Fellgiebel and Yakushev, 2011; Liu et al., 2011; Zhuang et al., 2012, 2013; Ito et al., 2015) have reported significant DTI changes in the fornix and hippocampal cingulum. It is well documented that the hallmarks of $\mathrm{AD}$ pathology begin in the transentorhinal region of the brain (Braak and Braak, 1991). Therefore, it may be there are greater structural white matter alterations in the hippocampus and MTL of patients with MCI and pre-clinical AD than patients with PTSD. Alternatively, the lack of PTSD-related DTI findings in the hippocampus and MTL may be attributable, as least in part, to the fact that most DTI studies of PTSD have only examined FA.

It has been suggested that other DTI parameters (i.e., axial and radial diffusivity) may be more sensitive to changes in axonal morphology and myelination than FA (Tyszka et al., 2006). For example, there is suggestive evidence that radial diffusivity is particularly sensitive to changes in myelination (Song et al., 2002, 2003, 2005). Because few DTI studies of PTSD have considered radial diffusivity, this may, at least in part, account for why past DTI studies of PTSD have not reported significant findings in the hippocampus or MTL. As a case in point, in a previous DTI study of veterans with and without PTSD, 18 of whom are part of the present report, our group did not find any significant medial temporal FA differences (Schuff et al., 2011). However, this study did not examine radial diffusivity. Thus, future studies will need to determine if vulnerability to trauma-related stress and PTSD has a similar effect on myelination in hippocampal white matter tracts as it appears to have in hippocampal gray matter.

In the central nervous system, myelin insulates and protects axons and facilitates the conduction of nerve impulses (Barkovich, 2000). Therefore, the loss of myelin can result in reduced nerve conduction velocity, reduced millisecond axonal precision, and reduced range of cortical synchrony. These, in turn, could impact the overall speed of mental processes, spike-timing dependent plasticity, and/or functional connectivity (Nave and Ehrenreich, 2014). However, having an excess of myelin, particularly in gray matter structures, may also have negative consequences. For example, there is evidence that myelin inhibits synapse formation and reduces plasticity in the central nervous system (Chen et al., 2000; McGee and Strittmatter, 2003; McGee et al., 2005), that oligodendrocytes inhibit axon growth cones (Fawcett et al., 1989; Bandtlow et al., 1990; Morganti et al., 1990), and that oligodendrocytes precursor cells are repulsive for growing axons (Chen et al., 2002a,b). Because the formation and elimination of axons and synapses are critical for learning and memory (Kleim et al., 2002, 2004; Holtmaat and Svoboda, 2009; Xu et al., 2009; Yang et al., 2009; Boele et al., 2013), one consequence of excessive myelin, particularly in the hippocampus, may be a reduced ability to learn and remember (Squire and Alvarez, 1995). In this context, it is noteworthy that memory disturbances are predominant in the presentation of PTSD (Samuelson, 2011) and are part of the diagnostic criteria (American Psychology Association, 2013).

The present findings should to be considered within the context of a number of limitations: First, the sample was small and these preliminary findings of increased hippocampal myelination in individuals with PTSD will need to be replicated in a larger sample. Second, the inclusion of only male veterans may limit generalizability of the findings. Third, the Glasser and Van Essen model, which depends on a relatively simplistic model of bound and unbound water, may not be unambiguously specific for myelin. Fourth, we did not perform a surface-based analyses of the $\mathrm{T} 1 \mathrm{w} / \mathrm{T} 2 \mathrm{w}$ ratio data as described by Glasser and Van Essen (2011) because we were primarily interested in examining myelination in the hippocampus. Thus, future research will need to investigate whether vulnerability to stress promotes myelin changes in other brain regions that have been implicated in PTSD (i.e., amygdala, anterior cingulate, insula, and orbitofrontal cortex; Rauch et al., 2006; Shin et al., 2006). Also, because PTSD has been associated with white matter alterations (Daniels et al., 2013), future investigations should examine/compare myelin changes in gray and white matter using $\mathrm{T} 1 \mathrm{w} / \mathrm{T} 2 \mathrm{w}$ ratio and radial diffusivity, which has been proposed be sensitive to alterations in myelination (Song et al., 2002, 2003, 2005). Fifth, we cannot be certain of the specificity the present findings to PTSD because many of the PTSD subjects had comorbid depression. However, assembling a PTSD group that is free of depressive symptoms is unlikely to generalize. Furthermore, there has been evidence that calls into question whether PTSD and depression are distinct entities among individuals exposed to trauma given the common criterion symptoms (Elhai et al., 2011). Other limitations include potential partial volume effects and the cross-sectional nature of the study, which limits our ability to determine causality and the subjective nature of patient history and clinical scores, which may be biased by under- or over-reporting. These limitations 
notwithstanding, the current findings, if replicated, suggest that stress-induced myelin formation in the hippocampus may be a potential mechanism underlying the structural and functional brain abnormalities associated with vulnerability to stress.

\section{REFERENCES}

Alexander, A. L., Lee, J. E., Lazar, M., and Field, A. S. (2007). Diffusion tensor imaging of the brain. Neurother. 3, 316-329. doi: 10.1016/j.nurt.2007.05.011

Almazan, G., Honegger, P., Du Pasquier, P., and Matthieu, J. M. (1986). Dexamethasone stimulates the biochemical differentiation of fetal forebrain cells in reaggregating cultures. Dev. Neurosci. 8, 14-23. doi: 10.1159/000112237

American Psychology Association. (2013). Diagnostic and Statistical Manual of Mental Disorders. Washington, D.C.

Antonow-Schlorke, I., Helgert, A., Gey, C., Coksaygan, T., Schubert, H., Nathanielsz, P. W., et al. (2009). Adverse effects of antenatal glucocorticoids on cerebral myelination in sheep. Obstet. Gynecol. 113, 142-151. doi: 10. 1097/AOG.0b013e3181924d3b

Bandtlow, C., Zachleder, T., and Schwab, M. E. (1990). Oligodendrocytes arrest neurite growth by contact inhibition. J. Neurosci. 10, 3837-3848.

Barkovich, A. J. (2000). Concepts of myelin and myelination in neuroradiology. AJNR Am. J. Neuroradiol. 21, 1099-1109.

Barres, B. A., Lazar, M. A., and Raff, M. C. (1994). A novel role for thyroid hormone, glucocorticoids and retinoic acid in timing oligodendrocyte development. Development 120, 1097-1108.

Blake, D. D., Weathers, F. W., Nagy, L. M., Kaloupek, D. G., Gusman, F. D., Charney, D. S., et al. (1995). The development of a Clinican-Administered PTSD Scale. J. Trauma. Stress 8, 75-90. doi: 10.1002/jts.2490080106

Bock, N. A., Hashim, E., Kocharyan, A., and Silva, A. C. (2011). Visualizing myeloarchitecture with magnetic resonance imaging in primates. Ann. NY Acad. Sci. 1225, E171-E781. doi: 10.1111/j.1749-6632.2011.06000.x

Bock, N. A., Kocharyan, A., Liu, J. V., and Silva, A. C. (2009). Visualizing the entire cortical myelination pattern in marmosets with magnetic resonance imaging. J. Neurosci. Methods 185, 15-22. doi: 10.1016/j.jneumeth.2009.08.022

Boele, H. J., Koekkoek, S. K., De Zeeuw, C. I., and Ruigrok, T. J. (2013). Axonal sprouting and formation of terminals in the adult cerebellum during associative motor learning. J. Neurosci. 33, 17897-17907. doi: 10.1523/JNEUROSCI.051113.2013

Braak, H., and Braak, E. (1991). Neuropathological staging of Alzheimer-related changes. Acta Neuropathol. 82, 239-259. doi: 10.1007/bf00308809

Cheng, J. D., and de Vellis, J. (2000). Oligodendrocytes as glucocorticoids target cells: functional analysis of the glycerol phosphate dehydrogenase gene. J. Neurosci. Res. 59, 436-445. doi: 10.1002/(sici)10974547(20000201)59:3<436::aid-jnr19>3.0.co;2-z

Chen, M. S., Huber, A. B., van der Haar, M. E., Frank, M., Schnell, L., Spillmann, A. A., et al. (2000). Nogo-A is a myelin-associated neurite outgrowth inhibitor and an antigen for monoclonal antibody IN-1. Nature 403, 434-439. doi: 10 . 1038/35000219

Chen, Z. J., Negra, M., Levine, A., Ughrin, Y., and Levine, J. M. (2002a). Oligodendrocyte precursor cells: reactive cells that inhibit axon growth and regeneration. J. Neurocytol. 31, 481-495. doi: 10.1023/A:1025791 614468

Chen, Z. J., Ughrin, Y., and Levine, J. M. (2002b). Inhibition of axon growth by oligodendrocyte precursor cells. Mol. Cell. Neurosci. 20, 125-139. doi: 10. 1006/mcne.2002.1102

Chetty, S., Friedman, A. R., Taravosh-Lahn, K., Kirby, E. D., Mirescu, C., Guo, F., et al (2014). Stress and glucocorticoids promote oligodendrogenesis in the adult hippocampus. Mol. Psychiatry 19, 1275-1283. doi: 10.1038/mp.2013.190

Daniels, J. K., Lamke, J. P., Gaebler, M., Walter, H., and Scheel, M. (2013). White matter integrity and its relationship to PTSD and childhood trauma-a systematic review and meta-analysis. Depress Anxiety 30, 207-216. doi: 10. 1002/da.22044

Desarnaud, F., Bidichandani, S., Patel, P. I., Baulieu, E. E., and Schumacher, M. (2000). Glucocorticosteroids stimulate the activity of the promoters of peripheral myelin protein-22 and protein zero genes in Schwann cells. Brain Res. 865, 12-16. doi: 10.1016/s0006-8993(00)02130-2
Longitudinal research will be needed to better understand how myelin changes in gray and white matter brain structures throughout the course of PTSD and in response to treatment for PTSD.

Directorate for Deployment Health Support of the Special Assistant to the Under Secretary of Defense (Personnel and Readiness) for Gulf War Illness Medical Readiness and Military Deployments. US demolition operations at the Khamisiyah ammunition point (case narrative). (2002). Available online at: http://www.gulflink.osd.mil/library/kham_info.jsp

Douaud, G., Menke, R. A., Gass, A., Monsch, A. U., Rao, A., Whitcher, B., et al (2013). Brain microstructure reveals early abnormalities more than two years prior to clinical progression from mild cognitive impairment to Alzheimer's disease. J. Neurosci. 33, 2147-2155. doi: 10.1523/JNEUROSCI.4437-12.2013

Elhai, J. D., de Francisco Carvalho, L., Miguel, F. K., Palmieri, P. A., Primi, R., and Christopher Frueh, B. (2011). Testing whether posttraumatic stress disorder and major depressive disorder are similar or unique constructs. J. Anxiety Disord 25, 404-410. doi: 10.1016/j.janxdis.2010.11.003

Fawcett, J. W., Rokos, J., and Bakst, I. (1989). Oligodendrocytes repel axons and cause axonal growth cone collapse. J. Cell Sci. 92, 93-100.

Fellgiebel, A., and Yakushev, I. (2011). Diffusion tensor imaging of the hippocampus in MCI and early Alzheimer's disease. J. Alzheimers Dis. 26, 257-262. doi: 10.3233/JAD-2011-0001

Fields, R. D. (2008). White matter in learning, cognition and psychiatric disorders. Trends Neurosci. 31, 361-370. doi: 10.1016/j.tins.2008.04.001

First, M., Spitzer, R., Gobbon, M., and Williams, J. (1995). Structured Clinical Interview for DMS-IV. New York, NY: New York State Psychiatric Institute Biometrics Research Department.

Fischl, B., van der Kouwe, A., Destrieux, C., Halgren, E., Ségonne, F., Salat, D. H., et al. (2004). Automatically parcellating the human cerebral cortex. Cereb. Cortex 14, 11-22. doi: 10.1093/cercor/bhg087

Fukuda, K., Nisenbaum, R., Stewart, G., Thompson, W. W., Robin, L., Washko, R. M., et al (1998). Chronic multisymptom illness affecting Air Force veterans of the Gulf War. JAMA 280, 981-988. doi: 10.1001/jama.280.11.981

Geyer, S., Weiss, M., Reimann, K., Lohmann, G., and Turner, R. (2011). Microstructural parcellation of the human cerebral cortex - from brodmann's post-mortem map to in vivo mapping with high-field magnetic resonance imaging. Front. Hum. Neurosci. 5:19. doi: 10.3389/fnhum.2011.00019

Gibson, E. M., Purger, D., Mount, C. W., Goldstein, A. K., Lin, G. L., Wood, L. S., et al. (2014). Neuronal activity promotes oligodendrogenesis and adaptive myelination in the mammalian brain. Science 344:1252304. doi: 10 . $1126 /$ science. 1252304

Glasser, M. F., and Van Essen, D. C. (2011). Mapping human cortical area in vivo based on myelin content as revealed by T1- and T2-weighted MRI. J. Neurosci. 31, 11597-11616. doi: 10.1523/JNEUROSCI.2180-11.2011

Hakak, Y., Walker, J. R., Li, C., Wong, W. H., Davis, K. L., Buxbaum, J. D., et al. (2001). Genome-wide expression analysis reveals dysregulation of myelinatinrelated genes in chronic schizophrenia. Proc. Natl. Acad. Sci. U S A 98, 4746-4751. doi: 10.1073/pnas.081071198

Hamilton, M. (1967). Development of a rating scale for primary depressive illness. Br. J. Soc. Clin. Psychol. 6, 278-296. doi: 10.1111/j.2044-8260.1967.tb00530.x

Hildebrand, C., Remahl, S., Persson, H., and Bjartmar, C. (1993). Meylinated nerve fibres in the CNS. Prog. Neurobiol. 40, 319-384. doi: 10.1016/03010082(93)90015-k

Holtmaat, A., and Svoboda, K. (2009). Experience-dependent structural synaptic plasticity in the mammalian brain. Nat. Rev. Neurosci. 10, 647-658. doi: 10. 1038/nrn2699

Ito, K., Sasaki, M., Takahashi, J., Uwano, I., Yamashita, F., Higuchi, S., et al. (2015). Detection of early changes in the parahippocampal and posterior cingulum bundles during mild cognitive impairment by using high-resolution multiparametric diffusion tensor imaging. Psychiatry Res. 231, 346-352. doi: 10. 1016/j.pscychresns.2015.01.020

Jenkinson, M., Bannister, P. R., Brady, J. M., and Smith, S. M. (2002). Improved optimisation for the robust and accurate linear registration and motion correction of brain images. Neuroimage 17, 825-841. doi: 10.1006/nimg.2002. 1132 
Jones, D., Christiansen, K., Chapman, R., and Aggleton, J. (2013). Distinct subdivisions of the cingulum bundle revealed by diffusion MRI fibre tracking: implications for neuropsychological investigations. Neuropsychologia 51, 67-78. doi: 10.1016/j.neuropsychologia.2012.11.018

Kalus, P., Slotboom, J., Gallinat, J., Mahlberg, R., Cattapan-Ludewig, K., Wiest, R., et al. (2006). Examining the gateway to the limbic system with diffusion tensor imaging: the perforant pathway in dementia. Neuroimage 30, 713-720. doi: 10. 1016/j.neuroimage.2005.10.035

Kennis, M., van Rooij, S. J., Tromp do, P. M., Fox, A. S., Rademaker, A. R., Kahn, R. S., et al. (2015). Treatment outcome-related white matter differences in veterans with posttraumatic stress disorder. Neuropsychopharmacology 40, 2434-2442. doi: 10.1038/npp.2015.94

Kleim, J. A., Freeman, J. H. Jr., Bruneau, R., Nolan, B. C., Cooper, N. R., Zook, A., et al. (2002). Synapse formation is associated with memory storage in the cerebellum. Proc. Natl. Acad. Sci. U S A 99, 13228-13231. doi: 10.1073/pnas. 202483399

Kleim, J. A., Hogg, T. M., VandenBerg, P. M., Cooper, N. R., Bruneau, R., and Remple, M. (2004). Cortical synaptogenesis and motor map reorganization occur during late, but not early, phase of motor skill learning. J. Neurosci. 24, 628-633. doi: 10.1523/jneurosci.3440-03.2004

Knowles, W. D. (1992). Normal anatomy and neurophysiology of the hippocampal formation. J. Clin. Neurophysiol. 9, 252-263. doi: 10.1097/00004691199204010-00006

Kumar, S., Cole, R., Chiappelli, F., and de Vellis, J. (1989). Differential regulation of oligodendrocyte markers by glucocorticoids: post-transcriptional regulation of both proteolipid protein and myelin basic protein and transcriptional regulation of glycerol phosphate dehydrogenase. Proc. Natl. Acad. Sci. U S A 86, 6807-6811. doi: 10.1073/pnas.86.17.6807

Le Bihan, D. (2003). Looking into the functional architecture of the brain with diffusion MRI. Nat. Rev. Neurosci. 4, 469-480. doi: 10.1038/nrn1119

Liu, Y., Spulber, G., Lehtimäki, K. K., Könönen, M., Hallikainen, I., Gröhn, H., et al. (2011). Diffusion tensor imaging and tract-based spatial statistics in Alzheimer's disease and mild cognitive impairment. Neurobiol. Aging 32, 1558-1571. doi: 10.1016/j.neurobiolaging.2009.10.006

Long, P., and Corfas, G. (2014). Dynamic regulation of myelination in health and disease. JAMA Psychiatry 71, 1296-1297. doi: 10.1001/jamapsychiatry.2014. 1049

Mann, S. A., Versmold, B., Marx, R., Stahlhofen, S., Dietzel, I. D., Heumann, R., et al (2008). Corticosteroids reverse cytokine-induced block of survival and differentiation of oligodendrocyte progenitor cells from rats. J. Neuroinflammation 5:39. doi: 10.1186/1742-2094-5-39

Masters, J. N., Finch, C. E., and Nichols, N. R. (1994). Rapid increase in glycerol phosphate dehydrogenase mRNA in adult rat brain: a glucocorticoiddependent stress response. Neuroendocrinology 60, 23-35. doi: 10 . $1159 / 000126716$

McGee, A. W., and Strittmatter, S. M. (2003). The Nogo-66 receptor: focusing myelin inhibition of axon regeneration. Trends Neurosci. 26, 193-198. doi: 10. 1016/s0166-2236(03)00062-6

McGee, A. W., Yang, Y., Fischer, Q. S., Daw, N. W., and Strittmatter, S. M. (2005). Experience-driven plasticity of visual cortex limited by myelin and Nogo receptor. Science 309, 2222-2226. doi: 10.1126/science. 1114362

Mielke, M. M., Okonkwo, O. C., Oishi, K., Mori, S., Tighe, S., Miller, M. I., et al. (2012). Fornix integrity and hippocampal volume predict memory decline and progression to Alzheimer's disease. Alzheimers Dement. 8, 105-113. doi: 10. 1016/j.jalz.2011.05.2416

Morganti, M. C., Taylor, J., Pesheva, P., and Schachner, M. (1990). Oligodendrocyte-derived J1-160/180 extracellular matrix glycoproteins are adhesive or repulsive depending on the partner cell type and time of interaction. Exp. Neurol. 109, 98-110. doi: 10.1016/s0014-4886(05)80012-3

Morris, J. C., Storandt, M., Miller, J. P., McKeel, D. W., Price, J. L., Rubin, E. H., et al. (2001). Mild cognitive impairment represents early-stage Alzheimer disease. Arch. Neurol. 58, 397-405. doi: 10.1001/archneur.58.3.397

Nave, K. A., and Ehrenreich, H. (2014). Myelination and oligodendrocyte functions in psychiatric diseases. JAMA Psychiatry 71, 582-584. doi: 10 . 1001/jamapsychiatry.2014.189

Nieuwenhuys, R. (2013). The myeloarchitectonic studies on the human cerebral cortex of the Vogt-Vogt school and their significance for the interpretation of functional neuroimaging data. Brain Struct. Funct. 218, 303-352. doi: 10. 1007/s00429-012-0460-z

Nowrangi, M. A., and Rosenberg, P. B. (2015). The fornix in mild cognitive impairment and Alzheimer's disease. Front. Aging Neurosci. 7:1. doi: 10. 3389/fnagi.2015.00001

Rauch, S. L., Shin, L. M., and Phelps, E. A. (2006). Neurocircuitry models of posttraumatic stress disorder and extinction: human neuroimaging research past, present and future. Biol. Psychiatry 60, 376-382. doi: 10.1016/j.biopsych. 2006.06.004

Rogalski, E. J., Murphy, C. M., deToledo-Morrell, L., Shah, R. C., Moseley, M. E., Bammer, R., et al (2009). Changes in parahippocampal white matter integrity in amnestic mild cognitive impairment: a diffusion tensor imaging study. Behav. Neurol. 21, 51-61. doi: 10.3233/BEN-2009-0235

Samuelson, K. (2011). Post-traumatic stress disorder and declarative memory functioning: a review. Dialogues Clin. Neurosci. 13, 346-351.

Schoenfeld, T. J., and Gould, E. (2012). Stress, stress hormones and adult neurogenesis. Exp. Neurol. 233, 12-21. doi: 10.1016/j.expneurol.2011. 01.008

Schuff, N., Zhang, Y., Zhan, W., Lenoci, M., Ching, C., Boreta, L., et al (2011). Patterns of altered cortical perfusion and diminished subcortical integrity in posttraumatic stress disorder: an MRI study. Neuroimage 54, S62-S68. doi: 10. 1016/j.neuroimage.2010.05.024

Sherin, J. E., and Nemeroff, C. B. (2011). Post-traumatic stress disorder: the neurobiological impact of psychological trauma. Dialogues Clin. Neurosci. 13, 263-278.

Shin, L. M., Rauch, S. L., and Pitman, R. K. (2006). Amydala, medial prefrontal cortex and hippocampal function in PTSD. Ann. N Y Acad. Sci. 1071, 67-79. doi: 10.1196/annals.1364.007

Song, S. K., Sun, S. W., Ju, W. K., Lin, S. J., Cross, A. H., and Neufeld, A. H. (2003). Diffusion tensor imaging detects and differentiates axon and myelin degeneration in mouse optic nerve after retinal ischemia. Neuroimage 20, 1714-1722. doi: 10.1016/j.neuroimage.2003.07.005

Song, S. K., Sun, S. W., Ramsbottom, M., Chang, C., Russell, J., and Cross, A. H. (2002). Dysmyelination revealed through MRI as increased radial (but unchanged axial) diffusion of water. Neuroimage 17, 1429-1436. doi: 10. 1006/nimg.2002.1267

Song, S. K., Yoshino, J., Le, T. Q., Lin, S. J., Sun, S. W., Cross, A. H., et al. (2005). Demyelination increases radial diffusivity in corpus callosum of mouse brain. Neuroimage 26, 132-140. doi: 10.1016/j.neuroimage.2005.01.028

Squire, L. R., and Alvarez, P. (1995). Retrograde amnesia and memory consolidation: a neurobiological perspective. Curr. Opin. Neurobiol. 5, 169-177. doi: 10.1016/0959-4388(95)80023-9

Tsuneishi, S., Takada, S., Motoike, T., Ohashi, T., Sano, K., and Nakamura, H. (1991). Effects of dexamethasone on the expression of myelin basic protein, proteolipid protein and glial fibrillary acidic protein genes in developing rat brain. Brain Res. Dev. Brain Res. 61, 117-123. doi: 10.1016/01653806(91)90121-x

Tyszka, J. M., Readhead, C., Bearer, E. L., Pautler, R. G., and Jacobs, R. E. (2006) Statistical diffusion tensor histology reveals regional dysmyelination effects in the shiverer mouse mutant. Neuroimage 29, 1058-1065. doi: 10.1016/j. neuroimage.2005.08.037

Von Der Heide, R. J., Skipper, L. M., Klobusicky, E., and Olson, I. R. (2013). Dissecting the uncinate fasciculus: disorders, controversies and a hypothesis. Brain 136, 1692-1707. doi: 10.1093/brain/awt094

Wolfe, J., Kimerling, R., Brown, P., Chresman, K., and Levin, K. (1996). "Psychometric review of the life stressor checklist-revised," in Instrumentation in Stress, Trauma and Adaptation, ed. B. H. Stamm (Lutherville, MD: Sidran Press), 144-151.

Xu, T., Yu, X., Perlik, A. J., Tobin, W. F., Zweig, J. A., Tennant, K., et al. (2009). Rapid formation and selective stabilization of synapses for enduring motor memories. Nature 462, 915-919. doi: 10.1038/nature08389

Yang, G., Pan, F., and Gan, W. B. (2009). Stably maintained dendritic spines are associated with lifelong memories. Nature 462, 920-924. doi: 10. 1038/nature08577

Yassa, M. A., Muftuler, L. T., and Stark, C. E. (2010). Ultrahigh-resolution microstructural diffusion tensor imaging reveals perforant path degradation in aged humans in vivo. Proc. Natl. Acad. Sci. U S A 107, 12687-12691. doi: 10 1073/pnas.1002113107 
Yoshiura, T., Higano, S., Rubio, A., Shrier, D. A., Kwok, W. E., Iwanaga, S., et al. (2000). Heschl and superior temporal gyri: low signal intensity of the cortex on T2-weighted MR images of the normal brain. Radiology 214, 217-221. doi: 10. 1148/radiology.214.1.r00ja17217

Young, K. M., Psachoulia, K., Tripathi, R. B., Dunn, S. J., Cossell, L., Attwell, D., et al. (2013). Oligodendrocyte dynamics in the healthy adult CNS: evidence for myelin remodeling. Neuron 77, 873-885. doi: 10.1016/j.neuron.2013.01.006

Zhu, W., Wiggins, R. C., and Konat, G. W. (1994). Glucocorticoid-induced upregulation of proteolipid protein and myelin-associated glycoprotein genes in C6 cells. J. Neurosci. Res. 37, 208-212. doi: 10.1002/jnr.490370206

Zhuang, L., Sachdev, P. S., Trollor, J. N., Reppermund, S., Kochan, N. A., Brodaty, H., et al. (2013). Microstructural white matter changes, not hippocampal atrophy, detect early amnestic mild cognitive impairment. PLos One 8:e58887. doi: 10.1371/journal.pone.0058887

Zhuang, L., Wen, W., Trollor, J. N., Kochan, N. A., Reppermund, S., Brodaty, H., et al. (2012). Abnormalities of the fornix in mild cognitive impairment are related to episodic memory loss. J. Alzheimers Dis. 29, 629-639. doi: 10. 3233/JAD-2012-111766

Conflict of Interest Statement: Dr. Neylan has consulted for Genentech and received study medication from Actelion for a trial funded by the Department of Defense and study medication from Glaxo Smith Kline for a trial funded by the Department of Veterans Affairs. The remaining authors declare no conflicts of interest.

Copyright (C) 2015 Chao, Tosun, Woodward, Kaufer and Neylan. This is an openaccess article distributed under the terms of the Creative Commons Attribution License (CC BY). The use, distribution and reproduction in other forums is permitted, provided the original author(s) or licensor are credited and that the original publication in this journal is cited, in accordance with accepted academic practice. No use, distribution or reproduction is permitted which does not comply with these terms. 